Opinion Article

Volume 1 - Issue 2

\title{
Abutment and Orthodontics for Endurance of Dental Crowns
}

\section{Ramya Chowdary"}

Dental Practitioner, India

*Corresponding author: Ramya Chowdary, Dental Practitioner, India

Received date: 11 December, 2021 | Accepted date: 18 December, 2021 |

Published date: 20 December, 2021

Citation: Chowdary R. (2021) Abutment and Orthodontics for Endurance of Dental Crowns. J Dent Oral Epidemiol 1(2). doi https://doi.org/10.54289/JDOEOp1

Copyright: () 2021 Chowdary R. This is an open-access article distributed under the terms of the Creative Commons Attribution License, which permits unrestricted use, distribution, and reproduction in any medium, provided the original author and source are credited.

Keywords: Botulinum Toxin; Monolithic zirconia; Osseointegration; Abutment, Myofascial Pain.

Abbreviations: OSA: Obstructive sleep apnea, BT: Botulinum Toxin, SLE: Systemic Lupus Erythematosus, SHP: Split-Increment Horizontal Placement.

\section{Opinion:}

The split-increment horizontal placement (SHP) is the popular technique being used, along with other therapeutic modus operandi. As more than adequate, SHP methods are being enhanced in barricade cavities for reducing the shrinkage stress that were generated during light polymerization. Such stress, if released unrestrained, may redress within the composite, tooth or at the adhesive contact [1]. The use of Botulinum Toxin (BT) in surgeries become idiomatic, as it is effective in reducing myofascial pain and improving its tasks over time. Thus, the the use of BT to most of the treatments reduce the potency of myofascial pain especially in adult patients [2]. Monolithic zirconia restorations have combined better esthetics and strength than other tooth-colored restorations, this zirconia offers superior robustness and endurance for the dental crowns making them attractive and expedient choice by dentists and patients (Figure-1). The tested frameworks and strategies of erbiumbased lasers are out of harm's way for removing or to in adhesive the ceramic brackets [3].

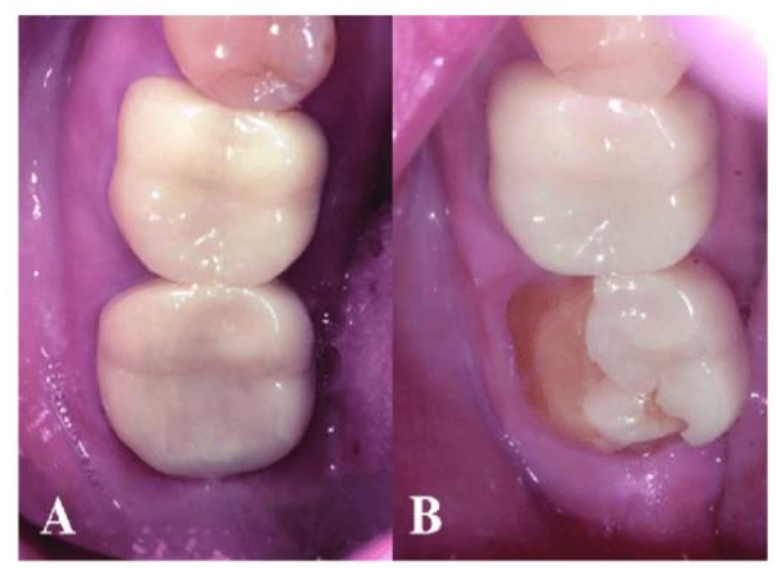

Figure 1: Monolithic zirconia teeth are more durable than the normal teeth as the enamel wears away.

Obstructive sleep apnea (OSA) is a disorder in which a person frequently stops breathing during sleep, OSA to be handled efficiently by diagnosing. [4]. OSA is treated with nasal
CPAP therapy, which is diagnosed by dentists and ENTspecialists. Any omissions during therapy effets the dental endurance. Osseointegration is defined as the direct 
structural and functional connection between the living bone and surface of a load-bearing implant. It is dominant for the triumph of teeth and bone implants [5]. During studies on healing and anchorage it is evalued that stability of titanium tooth root implants or fixtures are more durable as compared to others. The patients using non-conventional treatments to treat oral ulcer suffered side effects. [6]. Oral problems including tooth leads to dental frail. People suffered and suffering from SARS-CoV-2 are most striking novel clinical presentation was intraoral painful blisters or mouth ulcers mostly seen on the cheek mucosa [7].

In findings, Systemic Lupus Erythematosus (SLE) patients significantly have a greater number of missing teeth (Figure2). For teeth durability, it is mandatory to diagnosis, as the exact mechanism through which SLE patients experience periodontal breakdown and increased tooth loss is an avenue that warrants future research [8].

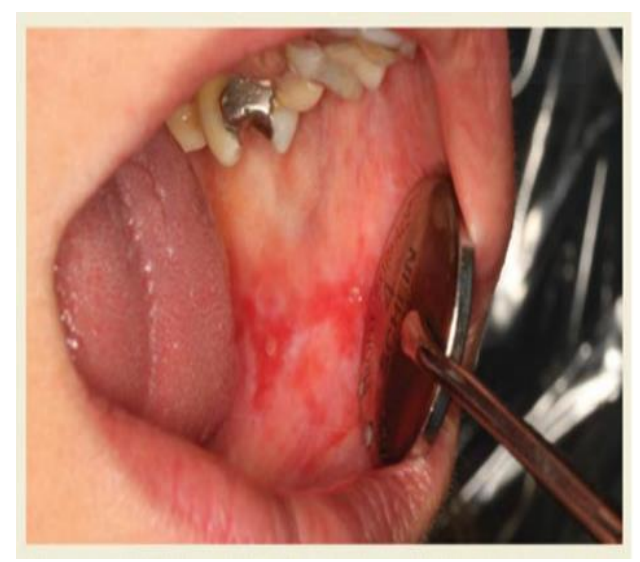

Figure 2: SLE patients also experience breakdown and increased tooth loss.

The patients who were diagnoses of SLE were also undergone for treatment and higher of the results undergone monolithic zirconia surgeries. Lots of research is going on to avoid ridge augmentation and multiple surgical procedures. Basal implants are dental implants that employ the basal cortical portion of jaws for the implant retention.
Abutment is the connector, the metal part which sereves as base for the crown. This is used in the osseointegrated as a fixed bridge, for the partial removal dentures and in implant

(Figure-3).

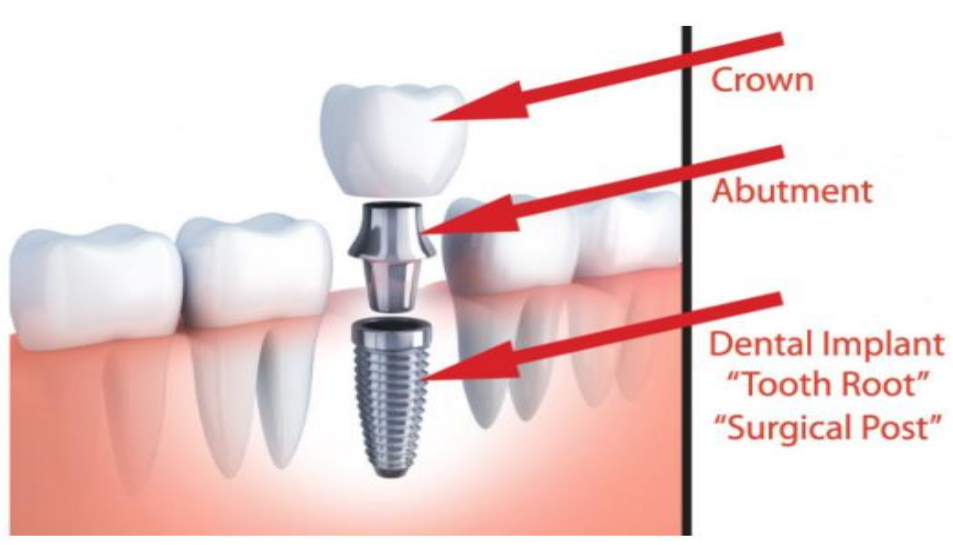

Figure 3: Abutment connecting both the crown and tooth root during implanting.

The patients with occlusal cavities undergo for osseointegration where BT used during surgeries, as the toxin plays a crucial role in reducing myofascial pain.

\section{Conclusion:}

During osseointegration, abutment plays a crucial role in connecting the crown and implant tooth root where, BT and 
monolithic zirconia are pivotal during therapies and implantation for the endurance of dental and jaw.

\section{References:}

1. Hassan KA, Khier SE (2021) Formation of Diagonal Gaps as Stress-Relieving Sites: Rethinking the Concept of Increment Splitting in Direct Occlusal Composite Restorations. J Dent Oral Epidemiol. 1(1).

2. Wambier LM, Petinati MFP, Corso P, Andrade CF, Larocca de Geus J, et al. (2021) Does the Use of Botulinum Toxin Reduce the Intensity of Myofascial Pain in Adult Patients? A Systematic Review and MetaAnalysis. J Dent Oral Epidemiol 1(2).

3. Raymond H, Corby LR, Marocho SMS (2021) Thermal Changes following Er,Cr: Ysgg Laser Irradiation through A Translucent Zirconia. J Dent Oral Epidemiol 1(1).

4. Srivastava P, Devi LM, Chauhan V. (2021) Treatment with Oral Appliances in Obstructive Sleep Apnea. J Dent Oral Epidemiol 1(2).
5. Nobles KP, Pal P, Janorkar AV, Williason RS. (2021) PEEK as a Potential Material for Dental Implants and its Biomechanical Properties and Osteoblast Cell Response. J Dent Oral Epidemiol 1(2).

6. Abdelnaser AG, Mahmoud EA, Ibrahium MZ, Gaafar S. (2021) What do Patients Use for Treating Their Oral Ulcers? And How do these Treatments Affect Their Quality of Life? J Dent Oral Epidemiol 1(1).

7. Baban B, Stevens MR, Ghasemi S, Boojar FMA, Dashti M (2021) New manifestation of Covid-19 in oral region, a potential faster diagnosis approach, a mini review. J Dent Oral Epidemiol 1(1).

8. Wu J.Y.W, Molli VLP, Hsiao C (2021) Periodontitis Risk in Patients with and Without Systemic Lupus Erythematosus: A Retrospective Study. J Dent Oral Epidemiol 1(1). 